\title{
Chidamide combined with cyclophosphamide, epirubicin, vindesine, prednisone, and etoposide (C-CHOEP) regimen is superior to the GDP-ML and CHOEP regimen for patients with newly diagnosed peripheral T-cell lymphoma
}

chong wei

Peking Union Medical College Hospital https://orcid.org/0000-0001-9690-8555

\section{Yan Zhang}

Peking Union Medical College Hospital

\section{Wei Wang}

Peking Union Medical College Hospital

\section{Lu Zhang}

Peking Union Medical College Hospital

\section{Yueying Mao}

Peking Union Medical College Hospital

\section{Jun Feng}

Peking Union Medical College Hospital

\section{Huacong Cai}

Peking Union Medical College Hospital

Wei Zhang ( $\approx$ vv1223@vip.sina.com )

https://orcid.org/0000-0001-9489-5965

\section{Daobin Zhou}

Peking Union Medical College Hospital

\section{Research article}

Keywords: peripheral T-cell lymphoma, chidamide, chemotherapy, treatment response

Posted Date: July 21st, 2020

DOI: https://doi.org/10.21203/rs.3.rs-46062/v1

License: (9) (1) This work is licensed under a Creative Commons Attribution 4.0 International License. Read Full License 


\section{Abstract \\ Background}

Outcomes with CHOP (cyclophosphamide, epirubicin, vindesine, and prednisone) or CHOP-like regimens are poor in most types of peripheral T-cell lymphomas (PTCLs). In this study, we investigated whether the combination of chidamide with CHOEP (cyclophosphamide, epirubicin, vindesine, etoposide, and prednisone) regimen (C-CHOEP) was superior to CHOEP and GDP-ML (gemcitabine, cisplatin, dexamethasone, methotrexate, and pegaspargase) regimen for previously untreated PTCL patients.

\section{Methods}

Between January 2012 and August 2019, a total of 94 newly diagnosed PTCL patients were included in this study, including 34 patients from the C-CHOEP phase 2 trial, 34 patients treated with CHOEP regimen matched to those in the C-CHOEP group, and 26 patients from a previous phase 2 trial of GDP-ML therapy.

\section{Results}

The complete remission rate of patients in the C-CHOEP group was significantly higher than that of GDP-ML and CHOEP group (55.9\% vs. $32.0 \%$ and $29.4 \%, p=0.047)$, with an overall response rate of 82.4 vs. $64.7 \%$, and $72.0 \%$, respectively $(p=0.244)$. The overall survival of patients in the $\mathrm{C}$-CHOEP group was significantly superior than that of the GDP-ML and CHOEP group, with 1- and 3- year OS rates of $80.9 \%$ and $73.5 \%$ in the C-CHOEP group vs. $67.4 \%$ and $36.9 \%$ in the GDP-ML group, and $64.6 \%$ and $48.9 \%$ in the CHOEP group $(p=0.048)$. Progression-free survival (PFS) was also improved with median PFS of 19.8 months in the C-CHOEP group vs. 6.6 and 8.1 months in the GDP$\mathrm{ML}$ and CHOEP group. The most common adverse events were hematologic toxicities. Grade 3-4 neutropenia and febrile neutropenia occurred in $58.8 \%$ and $20.6 \%$ of patients in the C-CHOEP group with no significant difference compared with the CHOEP group.

\section{Conclusion}

Our study suggested that C-CHOEP therapy was superior to CHOEP and GDP-ML therapy for newly diagnosed PTCL patients as shown by a significant improvement of $\mathrm{CR}$ rate and $\mathrm{OS}$ with a favorable safety profile.

\section{Trial registration:}

The study of C-CHOEP regimen is registered at ClinicalTrials.gov on December 2nd 2016 (NCT02987244). The study of GDP-ML regimen is registered at Chinese Clinical Trial Registry on March 30th 2012 (ChiCTR-ONC-12002055).

\section{Background}

Peripheral T cell lymphomas (PTCLs) are a heterogeneous group of mature T- and natural killer (NK) - cell neoplasms characterized by poor prognosis and aggressive clinical behavior [1]. PTCLs accounts for 25 to $30 \%$ of all non-Hodgkin's lymphomas (NHL) in China, which is significantly higher than in western countries [1, 2]. A consensus on the first-line treatment for PTCL patients has not been reached. Anthracycline-based regimens such as 
CHOP or CHOP-like regimens remain the most commonly used and complete remission (CR) rates of around $50 \%$ have been reported $[3,4]$. However, such regimens failed to induce sustained remission with a long-term survival of only $30-40 \%$ in most types of PTCLs [1, 3-7].

Evidence of other more intensive chemotherapies being superior to $\mathrm{CHOP}$ is unfortunately not sufficient. The addition of etoposide to CHOP (CHOEP) might improve response rate and event-free survival (EFS) for younger patients (aged $\leq 60$ years) $[7,8]$. Non-anthracycline based regimens such as gemcitabine-based chemotherapy were explored in several studies for PTCL patients but still showed non-superior results compared to CHOP-like chemotherapy [9]. At our center, Zhang $Y$ et al. conducted a phase 2 study of GDP-ML regimen (combined gemcitabine, cisplatin, dexamethasone, methotrexate, and pegaspargase) in patients with newly diagnosed PTCLs, which showed an ORR of $55.4 \%$ and 2-year OS rate of $38.2 \%$ (registered with www. chictr.org.cn, ChiCTR-ONC12002055) [10].

New drugs, including the histone deacetylase (HDAC) inhibitors romidepsin and belinostat, were approved for patients with relapsed or refractory PTCL in recent years [11, 12]. Chidamide is an innovative class I HDAC inhibitor independently developed in China and was approved by the China Food and Drug Administration (CFDA) for the treatment of relapsed or refractory PTCL [13]. Chidamide monotherapy had been assessed in a multicenter phase 2 trial and real-world study in China, with an ORR of 28-39\% in relapsed or refractory PTCL patients [14-15]. HDAC inhibitor combined with chemotherapy may be a new treatment choice for PTCL patients. Combination of chidamide with CHOEP regimen (C-CHOEP) in newly diagnosed PTCL patients was accessed in a phase 2 study at our center (registered with ClinicalTrials.gov, NCT02987244).

In this study, we aimed to evaluate the efficacy and safety of C-CHOEP regimen in newly diagnosed PTCL patients. Results were compared with CHOEP and GDP-ML regimen at our center. Given the heterogeneity in response and survival across different PTCL subtypes, we focused only on the three major subtypes of PTCLs, including peripheral T-cell lymphoma, not otherwise specified (PTCL, NOS), anaplastic large cell lymphoma (ALCL), ALKnegative, and angioimmunoblastic T-cell lymphoma (AITL).

\section{Methods}

\section{Patients}

Between January 2012 and August 2019, a total of 94 newly diagnosed PTCL patients who were treated in Peking Union Medical College Hospital were identified and included in this retrospective study. According to the first-line chemotherapy, patients were divided into 3 groups: C-CHOEP group, CHOEP group, and GDP-ML group. The CCHOEP group included newly diagnosed PTCL patients who were treated in the C-CHOEP phase 2 trial from January 2015 to August 2019. During the same period, patients who were treated with CHOEP regimen were matched to those receiving $\mathrm{C}$-CHOEP regimen at a ratio of 1:1 via matched-pair analysis. The patients were matched for the following variables: age ( \pm 5 years), Ann Arbor stage (I/II vs. III/IV), and the proportion of ALK-negative ALCL. The GDP-ML group consisted of 26 newly diagnosed PTCL patients who were treated with GDP-ML therapy between January 2012 and December 2014 in a previous phase 2 study at our center.

The inclusion criteria of the 3 groups were as follows: 1) histological findings consistent with PTCL, NOS, ALKnegative ALCL, or AITL on the basis of World Health Organization (WHO) classification [16]; 2) age between 18 and 75 years old; 3 ) Eastern Cooperative Oncology Group (ECOG) performance status (PS) of 0 to $2 ; 4$ ) complete staging and work-up data available. All pathological data were reviewed by 2 independent pathologists to confirm the 
diagnosis in our hospital. Subtypes including ALK-positive ALTL, extranodal NK/T-cell lymphoma, nasal type (ENKTL), and primary cutaneous T-cell lymphoma were excluded from this study.

The clinical data included patient demographics, physical examination, ECOG PS score, complete blood count, serum lactate dehydrogenase (LDH) level, bone marrow aspiration and biopsy, and computed tomography (CT) or positron emission tomography/CT (PET/CT) scan. Patients were staged according to the Ann Arbor Staging System. Patients were risk-stratified according to two prognostic scores, the International Prognostic Index (IPI) [17] and the Prognostic Index for PTCL-U patients (PIT) [18].

This study was conducted in accordance with the Declaration of Helsinki. Study protocols were approved by the institutional review board of Peking Union Medical College Hospital. Written informed consent was obtained from all patients before the collection of patients' information.

\section{Treatment}

The regimen of CHOEP chemotherapy were as follows: cyclophosphamide $\left(750 \mathrm{mg} / \mathrm{m}^{2}\right.$ intravenously on day 1$)$, epirubicin (70 mg/m² intravenously on day 1$)$, vindesine (4 mg intravenously on day 1$)$, prednisone (100 mg/d orally on days $1-5)$, and etoposide (100 mg intravenously on days 1-3). The CHOEP regimen was repeated every 3 weeks. The regimen of GDP-ML therapy were as follows: gemcitabine $\left(1 \mathrm{~g} / \mathrm{m}^{2}\right.$ intravenously on day 1$)$, cisplatin $\left(75 \mathrm{mg} / \mathrm{m}^{2}\right.$ intravenously on day 1), dexamethasone (40 mg/d intravenously on days $1-4)$, methotrexate $\left(1 \mathrm{~g} / \mathrm{m}^{2}\right.$ intravenously for 4 hours on day 2), and pegaspargase (3750 U subcutaneous on day 5). The GDP-ML regimen was repeated every 4 weeks. Six cycles of CHOEP and GDP-ML therapy were planned for the protocol treatment.

The treatment of patients in the C-CHOEP group included an induction phase of 6 cycles of C-CHOEP therapy at a 3week interval followed by a maintenance phase of chidamide. During the induction phase, chidamide 20 mg twice weekly was added to CHOEP regimen and started on day 1 of the first cycle of CHOEP therapy. The dose and administration schedule of CHOEP therapy were the same as the patients in the CHOEP group. Chidamide could be discontinued during myelosuppressive period after CHOEP therapy until recovery. Patients who achieved PR or CR response after 6 cycles of C-CHOEP induction would receive chidamide maintenance treatment for 2 years. During the maintenance phase, chidamide $20 \mathrm{mg}$ twice weekly was administered continuously until disease progression, unacceptable toxicity, or completion of 2 years maintenance treatment. The dose of chidamide was reduced by $5 \mathrm{mg}$ in patients who had grade 3 non-hematologic or hematologic toxicities.

Patients who achieved CR or PR after chemotherapy in the 3 groups could undergo autologous stem-cell transplantation (auto-SCT). The decision was made according to the physician's discretion mainly on the basis of the patient's age, performance status, and the patient's willingness. Peripheral-blood stem cells were collected after 4 cycles, and the conditioning regimen consisted of carmustine, etoposide, cytarabine, and melphalan (BEAM).

\section{Assessment of efficacy}

Efficacy was evaluated after 2-4 cycles and 1 month after the completion of chemotherapy. Responses were assessed by CT scan of chest and abdomen or PET/CT and were classified as complete remission (CR), partial remission (PR), stable disease (SD) and progressive disease (PD) according to the 2007 Revised Response Criteria for Malignant Lymphoma [19]. If PET/CT scan was performed, responses were assessed according to published recommendations [20]. The overall response rate (ORR) was defined as the proportion of patients who achieved CR or PR as their best response. Toxicity was graded according to the Common Terminology Criteria for Adverse Events (CTCAE) version 4.0 . 


\section{Statistical analysis}

The primary end point was ORR. The secondary end points were overall survival (OS), progression-free survival (PFS), and toxicity. PFS was defined as time from diagnosis to the date of disease progression or death from any cause or the last follow-up. OS was defined as time from diagnosis to death from any cause or the last follow-up. Distribution differences of clinical characteristics between groups were analyzed with the Kruskall-Wallis test for age and $\chi^{2}$ test or if necessary Fisher exact test for the other variables. Survival analysis of OS and PFS was estimated using Kaplan-Meier method. Survival rates between groups were compared using the Log-rank test. $P$ value $<0.05$ was considered significant. All data analyses were performed using the SPSS 20.0 software (SPSS Inc., Chicago, IL, USA).

\section{Results}

Patient characteristics

The number of patients in the 3 groups were 26 cases in the GDP-ML group, 34 cases in the CHOEP group, and 34 cases in the C-CHOEP group. The baseline characteristics of patients in the 3 groups were listed in Table 1. The median age at diagnosis were 40 (range, 18-72) years in the GDP-ML group, 53 (range, 18-74) years in the CHOEP group, and 57 (range, 23-75) years in the C-CHOEP group. B symptoms was found in $69.2 \%, 76.4 \%$, and $67.6 \%$ of patients in the three groups and the elevation of LDH level was observed in $57.7 \%, 82.4 \%$, and $52.9 \%$ of the patients, respectively. At the time of diagnosis, $84.6 \%, 82.3 \%$, and $82.3 \%$ of patients were in stage III/IV. $46.2 \%, 55.9 \%$, and $44.1 \%$ of the patients were determined to be high-intermediate or high risk based on the IPI score, while the proportion of high-intermediate or high risk patients based on PIT score were $42.3 \%, 47.0 \%$, and $38.2 \%$, respectively.

When comparing demographic and clinical characteristics among the 3 groups, only a significant higher proportion of LDH elevation was found in the CHOEP group $(p=0.026)$. No other significant differences of clinical characteristics were found among the 3 groups. The proportion of different histologic subtypes also showed no significant differences among the 3 groups. 
Table 1

पComparison of clinical characteristics of patients in the 3 groups

\begin{tabular}{|lllll|}
\hline Characteristic & $\begin{array}{l}\text { GDP-ML group } \\
(\mathbf{n}=\mathbf{2 6})\end{array}$ & $\begin{array}{l}\text { CHOEP group } \\
(\mathbf{n}=\mathbf{3 4})\end{array}$ & $\begin{array}{l}\text { C-CHOEP group } \\
(\mathbf{n}=\mathbf{3 4})\end{array}$ & $P$ value \\
\hline Age, years & & & & \\
\hline Median (Range) & $40(18-72)$ & $53(18-74)$ & $57(23-75)$ & 0.052 \\
\hline \60 & $6(23.1)$ & $14(41.2)$ & $12(35.3)$ & 0.335 \\
\hline Sex, male & $16(61.5)$ & $20(58.9)$ & $14(41.2)$ & 0.209 \\
\hline Ann Arbor stage III/IV & $22(84.6)$ & $28(82.3)$ & $28(82.3)$ & 0.966 \\
\hline B symptoms present & $18(69.2)$ & $26(76.4)$ & $23(67.6)$ & 0.698
\end{tabular}

Elevated LDH

\begin{tabular}{|c|c|c|c|c|}
\hline Elevated LDH & $15(57.7)$ & $28(82.4)$ & $18(52.9)$ & 0.026 \\
\hline ECOG performance status $₫ 1$ & $11(42.3)$ & $10(29.4)$ & $9(26.5)$ & 0.716 \\
\hline Extranodal involvement $₫ 1$ & $10(38.5)$ & $17(50.0)$ & $11(32.4)$ & 0.324 \\
\hline Bone marrow involvement & $5(19.2)$ & $10(29.4)$ & $7(20.6)$ & 0.634 \\
\hline IPI score $\llbracket 2$ & $12(46.2)$ & $19(55.9)$ & $15(44.1)$ & 0.591 \\
\hline PIT score $₫ 1$ & $11(42.3)$ & $16(47.0)$ & $13(38.2)$ & 0.696 \\
\hline Histologic subtypes & & & & 0.085 \\
\hline PTCL, NOS & $16(61.5)$ & $14(41.2)$ & $10(29.4)$ & \\
\hline AITL & $5(19.2)$ & $12(35.3)$ & $16(47.1)$ & \\
\hline ALK-negative ALCL & $5(19.2)$ & $8(23.5)$ & $8(23.5)$ & \\
\hline \multicolumn{5}{|l|}{ Abbreviations: } \\
\hline
\end{tabular}

Response to treatment

The responses of the 3 groups were summarized in Table 2. Responses could be accessed in 25 patients in the GDPML group, 34 patients in the CHOEP group, and 34 patients in the C-CHOEP group. CR was achieved in $32.0 \%, 29.4 \%$, and $55.9 \%$ of patients in the GDP-ML, CHOEP, and C-CHOEP group with an ORR of $72.0 \%, 64.7 \%$, and $82.4 \%$, respectively. The $\mathrm{CR}$ rate of C-CHOEP group was significantly higher than that of CHOEP group and GDP-ML group 
$(p=0.047)$. The ORR was also higher in the C-CHOEP group. However, this difference did not achieve statistical significance $(p=0.244)$.

After C-CHOEP induction, 21 of the 28 responding patients in the C-CHOEP group received chidamide maintenance treatment, including 15 patients with CR response and 6 patients with PR response. The reasons for not receive chidamide maintenance of the responding patients were as follows: early relapse $(n=3)$ or disease progression $(n=$ 3 ) and died of pulmonary infection $(n=1)$. By the end of this study, 18 patients remained in chidamide maintenance treatment without disease relapse or progression (including 14 CR patients and 4 PR patients). With a median follow-up time of 33 (range, 1-59) months, the median duration of response (DOR) was not reached.

Table 2

Responses of the 3 groups

\begin{tabular}{|c|c|c|c|c|}
\hline \multirow[t]{2}{*}{ Response } & \multicolumn{3}{|c|}{ No. of Patients (\%) } & \multirow[b]{2}{*}{$P$ value } \\
\hline & GDP-ML group & CHOEP group & C-CHOEP group & \\
\hline No. of patients & 26 & 34 & 34 & \\
\hline Median number of cycles & 4 & 4.5 & 5.5 & \\
\hline $\mathrm{CR}$ & $8(32.0)$ & $10(29.4)$ & $19(55.9)$ & 0.047 \\
\hline PR & $10(40.0)$ & $12(35.3)$ & $9(26.5)$ & \\
\hline SD & $0(0)$ & $2(3.0)$ & $0(0)$ & \\
\hline PD & $7(28.0)$ & $10(30.3)$ & $6(17.6)$ & \\
\hline ORR & 72.0 & 64.7 & 82.4 & 0.244 \\
\hline Treatment-related death & $2(7.7)^{*}$ & $0(0)$ & $1(2.9)^{\#}$ & 0.594 \\
\hline Auto-SCT & 6 & 5 & 8 & 0.810 \\
\hline \multicolumn{5}{|c|}{$\begin{array}{l}\text { Abbreviations: CR, complete remission; PR, partial remission; PD, progressive disease; SD, stable disease; ORR, } \\
\text { overall response rate; auto-SCT, autologous stem-cell transplantation. }\end{array}$} \\
\hline \multicolumn{5}{|c|}{ *Including 1 patient with PR response. } \\
\hline
\end{tabular}

Long-term survival

The median follow-up time was 79 (range, 1-88) months, 45 (range, 1-65) months, and 33 (range, 1-59) months in the GDP-ML, CHOEP, and C-CHOEP group. By the end of the follow-up time, there were 16 deaths in the GDP-ML group (13 due to disease progression or relapse, 1 due to central nervous system (CNS) infection, 1 due to febrile neutropenia, and 1 due to secondary lung carcinoma), 16 deaths in the CHOEP group (all due to disease progression or relapse), and 8 deaths in the C-CHOEP group (7 due to disease progression or relapse and 1 due to pulmonary infection).

The median PFS of the GDP-ML, CHOEP, and C-CHOEP groups were 6.6, 8.1, and 19.8 months with 1- and 3- year PFS rates of $45.1 \%$ and $28.1 \%$ in the GDP-ML group, $42.7 \%$ and $33.7 \%$ in the CHOEP group, and $63.5 \%$ and $46.8 \%$ in the C-CHOEP group (Fig. 1a). Though a trend of better PFS was observed in the C-CHOEP group, this difference did not achieve statistical significance $(p=0.330)$. The median OS of the GDP-ML and CHOEP groups were 23.4 and 
31.5 months, while that of the C-CHOEP group was not reached. The 1- and 3-year OS rates were $67.4 \%$ and $36.9 \%$ in the GDP-ML group, $64.6 \%$ and $48.9 \%$ in the CHOEP group, and $80.9 \%$ and $73.5 \%$ in the C-CHOEP group (Fig. $1 \mathrm{~b}$ ). The patients in the C-CHOEP group presented significant superior survival outcomes over those in the GDP-ML and CHOEP group $(p=0.048)$.

After analyzing only the subgroup of responding patients, a trend of better PFS and OS was also found in the CCHOEP group than that of the CHOEP and GDP-ML group (3-year PFS rates: $58.6 \%$ in the C-CHOEP group vs. $40.8 \%$ and $45.9 \%$ in the GDP-ML and CHOEP group, $p=0.427$; 3-year OS rates: $81.7 \%$ vs. $50.4 \%$ and $68.7 \%$, respectively, $p=$ 0.166;) (Fig. 1c and 1d). The small sample size in each group could be a reason why none of the differences were statistically significant.

Figure 1 Kaplan-Meier curves of PFS for all patients (a) and responding patients (c) in the 3 groups, OS for all patients (b) and responding patients (d) in the 3 groups

Adverse events

Table 3 lists all grade 3-5 adverse events (AEs) that occurred in the three groups. The most common AE was hematologic toxicity. The incidence of grade $3-4$ neutropenia was $61.6 \%$ in the GDP-ML group, $55.9 \%$ in the CHOEP group, and $58.8 \%$ in the C-CHOEP group. Grade $3-5$ febrile neutropenia occurred in $34.5 \%, 20.6 \%$, and $20.6 \%$ of patients in the three groups with a total of 5 life-threatening infections requiring admission into intensive care unit, leading to 1 death (due to septic shock in GDP-ML group ). Although the incidence of febrile neutropenia was higher in the GDP-ML group, this difference did not achieve statistical significance $(p=0.367)$.

The non-hematologic toxicities were mainly presented in grade 1-2. The only grade 3-4 non-hematologic AE that occurred at an incidence of more than 10\% was ALT elevation in the GDP-ML group. Grade 5 infection occurred in 2 patient ( 1 case of CNS infection in the GDP-ML group and 1 case of pulmonary infection in the C-CHOEP group). Two patients (7.7\%) in the GDP-ML group experienced grade 3 acute renal injury. One patient (2.9\%) in the CHOEP group and 1 patient (2.9\%) in the C-CHOEP group had grade 3 allergy to etoposide. Chemotherapy of the 2 patients was adjusted to CHOP regimen in the subsequent courses. No EBV or hepatitis B virus (HBV) reactivation was observed in the three groups. There were no significant differences in the incidence of grade 3-4 AEs among the three groups.

During the chidamide maintenance phase, the majority of patients tolerated chidamide well. The most common AE was also hematologic toxicity. The grade 3-4 AEs included neutropenia $(n=2,9.5 \%)$, thrombocytopenia $(n=1$, $4.8 \%$ ), and anemia $(n=1,4.8 \%)$. Two patients had dose reduction of chidamide to $15 \mathrm{mg}$ due to AEs (including 1 patient due to neutropenia and 1 due to thrombocytopenia). 
Table 3

Adverse events of the three groups

\begin{tabular}{|c|c|c|c|c|c|c|c|c|c|c|}
\hline \multirow[t]{2}{*}{ Toxicity } & \multicolumn{3}{|c|}{ GDP-ML $(n=26)$} & \multicolumn{3}{|c|}{ CHOEP $(n=34)$} & \multicolumn{3}{|c|}{ C-CHOEP $(n=34)$} & \multirow{2}{*}{$\begin{array}{l}P \\
\text { value }\end{array}$} \\
\hline & $\begin{array}{l}\text { Grade } \\
3\end{array}$ & $\begin{array}{l}\text { Grade } \\
4\end{array}$ & $\begin{array}{l}\text { Grade } \\
5\end{array}$ & $\begin{array}{l}\text { Grade } \\
3\end{array}$ & $\begin{array}{l}\text { Grade } \\
4\end{array}$ & $\begin{array}{l}\text { Grade } \\
5\end{array}$ & $\begin{array}{l}\text { Grade } \\
3\end{array}$ & $\begin{array}{l}\text { Grade } \\
4\end{array}$ & $\begin{array}{l}\text { Grade } \\
5\end{array}$ & \\
\hline Neutropenia & $\begin{array}{l}6 \\
(23.1)\end{array}$ & $\begin{array}{l}10 \\
(38.5)\end{array}$ & $0(0)$ & $\begin{array}{l}9 \\
(26.5)\end{array}$ & $\begin{array}{l}10 \\
(29.4)\end{array}$ & $0(0)$ & $\begin{array}{l}7 \\
(20.6)\end{array}$ & $\begin{array}{l}13 \\
(38.2)\end{array}$ & $0(0)$ & 0.860 \\
\hline $\begin{array}{l}\text { Febrile } \\
\text { neutropenia }\end{array}$ & $\begin{array}{l}7 \\
(26.9)\end{array}$ & $\begin{array}{l}1 \\
(3.8)\end{array}$ & $\begin{array}{l}1 \\
(3.8)\end{array}$ & $\begin{array}{l}5 \\
(14.7)\end{array}$ & $\begin{array}{l}2 \\
(5.9)\end{array}$ & $0(0)$ & $\begin{array}{l}6 \\
(17.6)\end{array}$ & $\begin{array}{l}1 \\
(2.9)\end{array}$ & $0(0)$ & 0.376 \\
\hline Anemia & $\begin{array}{l}2 \\
(7.7)\end{array}$ & $0(0)$ & $0(0)$ & $\begin{array}{l}2 \\
(5.9)\end{array}$ & $0(0)$ & $0(0)$ & $\begin{array}{l}3 \\
(8.8)\end{array}$ & $0(0)$ & $0(0)$ & 0.736 \\
\hline Thrombocytopenia & $\begin{array}{l}2 \\
(7.7)\end{array}$ & $\begin{array}{l}1 \\
(3.8)\end{array}$ & $0(0)$ & $\begin{array}{l}2 \\
(5.9)\end{array}$ & $0(0)$ & $0(0)$ & $\begin{array}{l}3 \\
(8.8)\end{array}$ & $0(0)$ & $0(0)$ & 0.899 \\
\hline ALT elevation & $\begin{array}{l}3 \\
(11.5)\end{array}$ & $0(0)$ & $0(0)$ & $\begin{array}{l}1 \\
(2.9)\end{array}$ & $0(0)$ & $0(0)$ & $\begin{array}{l}1 \\
(2.9)\end{array}$ & $0(0)$ & $0(0)$ & 0.675 \\
\hline Acute renal injury & $\begin{array}{l}2 \\
(7.7)\end{array}$ & $0(0)$ & $0(0)$ & $0(0)$ & $0(0)$ & $0(0)$ & $0(0)$ & $0(0)$ & $0(0)$ & 0.074 \\
\hline Infection & $\begin{array}{l}1 \\
(3.8)\end{array}$ & $0(0)$ & $\begin{array}{l}1 \\
(3.8)\end{array}$ & $\begin{array}{l}1 \\
(2.9)\end{array}$ & $0(0)$ & $0(0)$ & $\begin{array}{l}1 \\
(2.9)\end{array}$ & $0(0)$ & $\begin{array}{l}1 \\
(2.9)\end{array}$ & 0.707 \\
\hline Allergy & $0(0)$ & $0(0)$ & $0(0)$ & $\begin{array}{l}1 \\
(2.9)^{*}\end{array}$ & $0(0)$ & $0(0)$ & $\begin{array}{l}1 \\
(2.9)^{\star}\end{array}$ & $0(0)$ & $0(0)$ & 1.000 \\
\hline Nausea/Vomiting & $\begin{array}{l}2 \\
(7.7)\end{array}$ & $0(0)$ & $0(0)$ & $\begin{array}{l}1 \\
(2.9)\end{array}$ & $0(0)$ & $0(0)$ & $\begin{array}{l}1 \\
(2.9)\end{array}$ & $0(0)$ & $0(0)$ & 0.645 \\
\hline \multicolumn{11}{|c|}{ Abbreviations: ALT, alanine aminotransferase. } \\
\hline
\end{tabular}

\section{Discussion}

PTCLs are relatively rare lymphomas with mostly poor outcomes. In contrast to the progress made in the treatment of aggressive B-cell lymphoma, management of patients with PTCL is disappointing, with no major progress made over the past decades. Due to the rarity and geographic variation of the disease, randomized prospective trials are scarce and there is no consensus on the optimal therapy for PTCL patients. CHOP or CHOP-like regimens are the most widely used first-line therapy. The CR rates of around $50 \%$ have been reported. However, with the exception of ALK-positive ALCL, most types of PTCLs exhibited poor long-term survival. In China, a retrospective study included a total of 679 PTCL patients over two decades. The 5-year OS rate of $34.9 \%$ in AITL and $27.6 \%$ in PTCL, NOS were reported in this study, with no improvement of survival during the past two decades [5].

Given the poor outcomes associated with $\mathrm{CHOP}$ as first-line therapy, other more intensive chemotherapies have been investigated. High-dose CHOP alternating with ESHAP (etoposide, cisplatin, cytarabine and prednisone) followed by auto-SCT in young patients failed to overcome the poor prognosis of PTCL patients. The CR rate of $49 \%$ and 4 -year PFS and OS rates of $30 \%$ and $39 \%$ were reported in this study [21]. In a retrospective study from MD Anderson Cancer Center, the CR rate of more intensive regimens, including hyper-CVAD (cyclophosphamide, vincristine, doxorubicin, and dexamethasone) was not superior to CHOP regimen, with CR rate of 59\% and 3-year OS rates of 
49\% [22]. Gemcitabine-based regimens also failed to show advantage over standard CHOP regimen. In a phase 2 multicentre randomized trial, the CR rate of GEM-P regimen (gemcitabine, cisplatin, and methylprednisolone) was inferior to CHOP regimen (46\% vs. 62\%), with 2-year PFS rates of 38\% versus $37 \%$, respectively [9]. The above studies suggested that the past usage of cytotoxic chemotherapy at varying intensity was not sufficient in the treatment of PTCL. There is a critical need for new treatment approaches.

Epigenetic dysregulation plays an important role in PTCL oncogenesis. In recent years, HDAC inhibitors have emerged as novel therapeutic agents for PTCL. Since 2006, three of the 7 new drugs approved by the US Food and Drug Administration (FDA) for the treatment of PTCL were HDAC inhibitors, including vorinostat, romidepsin and belinostat. In the phase 2 trial of romidepsin for patients with relapsed PTCL, an ORR of $38 \%$ was reported with median DOR of 8.9 months [11]. The BELIEF trial evaluated belinostat for patients with relapsed or refractory PTCL. An ORR of $25.8 \%$ and median DOR of 13.6 months were achieved [12]. The safety and efficacy of romidepsin combined with CHOP was assessed in previously untreated PTCL patients. A comparable CR rate (51\%) and more favorable PFS and OS rates (41.0\% and $70.7 \%$ at 30 months) compared with CHOP alone were achieved. However, this combination therapy was associated with severe toxicity, including $89 \%$ of grade $3-4$ neutropenia, $78 \%$ of grade 3-4 thrombocytopenia, and 3 cases of acute cardiac toxicity [23].

In our study, combining chidamide with CHOEP chemotherapy proved to be effective, with CR rate of $55.9 \%$ and ORR of $82.4 \%$. Several studies reported relatively high CR rate over $50 \%$ with CHOP or CHOEP therapy alone. In the study conducted by d'Amore $\mathrm{F}$ et al, $\mathrm{CR}$ rate of $51 \%$ and ORR of $82 \%$ were reported with $\mathrm{CHOP} / \mathrm{CHOEP}$ regimen [24]. However, the time interval of $\mathrm{CHOP} / \mathrm{CHOEP}$ was shorten to 2 weeks which is not feasible in elderly patients. In the phase 3 study evaluating brentuximab vedotin combined with chemotherapy for CD30-positive PTCL, CR rate of $56 \%$ and ORR of $72 \%$ were reported in the CHOP control group [25]. However, $22 \%$ of the enrolled patients in this study were ALK-positive ALCL with relatively good prognosis. Considering that the histologic subtypes were mainly AITL and PTCL, NOS with no ALK-positive ALCL included and more than 1/3 of patients were older than 60 years in our study, the response achieved with $\mathrm{C}$-CHOEP regimen was satisfactory. The significantly higher $\mathrm{CR}$ rate compared with CHOEP therapy further confirmed the superiority of C-CHOEP therapy.

Although a fair number of PTCL patients are sensitive to chemotherapy, their response duration is often short with frequent relapse resulting in poor long-term survival. The relapse rate of $27-43 \%$ was reported in PTCL patients, generally higher than that of B-cell lymphoma [26-27]. In our study, the median DOR was not reached with a median follow-up time of 33 months. Patients in the C-CHOEP group showed much superior long-term survival (3-year PFS and OS rates of $46.8 \%$ and $73.5 \%$ ) than the $30-40 \%$ long-term survival reported with CHOP regimen in previous studies. These results indicated a more durable response achieved with $\mathrm{C}$-CHOEP induction and chidamide maintenance.

In general, chidamide monotherapy had a very favorable toxicity profile. In the real-world study of chidamide in relapsed or refractory PTCL, AEs of grade 3 or higher were mainly hematologic toxicities including thrombocytopenia (10\%) and neutropenia (6\%) [15]. In our study, combination of chidamide with CHOEP regimen was well tolerated with no additional hematologic toxicities and increased risk of infections compared to the CHOEP regimen. Chidamide maintenance was also feasible with mostly mild hematologic toxicities. In a pilot study evaluating romidepsin in patients with ENKTL, the trial was discontinued due to severe Epstein-Barr virus (EBV) reactivation in 3 of the 5 enrolled patients [28]. No EBV or HBV reactivation was observed either in the C-CHOEP induction phase or the chidamide maintenance phase in our study. 
The limitations of our study also need to be acknowledged. Due to the relatively small number of PTCL patients in a single institution, conducting randomized controlled trial was relatively difficult and historic groups were used as controls in our study. The study results may be confounded by selection bias, unbalanced demographics, and time differences. However, clinical experience of our study may serve as reference for future multicentre phase 3 randomized controlled trials. Differences in responses and survivals of different histologic subtypes of PTCL could also be investigated in further large-scale studies.

\section{Conclusion}

In conclusion, this study demonstrate that chidamide combined with CHOEP therapy is effective and well-tolerated for newly diagnosed PTCL patients. A significant higher CR rate and prolonged OS was obtained in the C-CHOEP group compared with GDP-ML and CHOEP group. Chidamide maintenance might contribute to a relatively low relapse rate and prolonged survival of the responding patients. However, these conclusions need to be further verified in large-scale multicenter studies and randomized controlled clinical trials.

\section{Abbreviations}

PTCL: peripheral T-cell lymphoma; NHL: non-Hodgkin's lymphoma; CHOP: cyclophosphamide, epirubicin, vindesine, and prednisone; CHOEP: cyclophosphamide, epirubicin, vindesine, etoposide, and prednisone; GDP-ML: gemcitabine, cisplatin, dexamethasone, methotrexate, and pegaspargase; HDAC: histone deacetylase; WHO: World Health Organization; CFDA: China Food and Drug Administration; PTCL, NOS: peripheral T-cell lymphoma, not otherwise specified; ALCL: anaplastic large cell lymphoma; AITL: angioimmunoblastic T-cell lymphoma; ENKTL: extranodal NK/T-cell lymphoma, nasal type; ECOG: Eastern Cooperative Oncology Group; LDH: lactate dehydrogenase; EBV: Epstein-Barr virus; CT: computed tomography; PET: positron emission tomography; IPI: International Prognostic Index; PIT: Prognostic Index for PTCL-U patients; auto-SCT: autologous stem-cell transplantation; BEAM: carmustine, etoposide, cytarabine, and melphalan; CR: complete remission; PR: partial remission; SD: stable disease; PD:

progressive disease; CTCAE: Common Terminology Criteria for Adverse Events; OS: overall survival; PFS: progression-free survival; EFS: event-free survival; AE: adverse events;

\section{Declarations}

\section{Ethics approval and consent to participate}

The study was done in accordance with the Declaration of Helsinki. The protocol was approved by the institutional review board of Peking Union Medical Hospital. Informed consent was obtained from all individual participants included in the study.

\section{Consent for publication}

Not applicable.

\section{Availability of data and materials}

The datasets used and analyzed during the current study are available from the corresponding author on reasonable request.

\section{Competing interests}

Page $11 / 14$ 
The authors declare that they have no conflict of interest.

\section{Funding}

National Natural Science Foundation of China (NSFC) (No.81970188)『CAMS Innovation Fund for Medical Sciences (CIFMS) (No. 2016-12M-1-001), and Natural Science Foundation of Beijing Municipality (No. 7202154).

\section{Authors' contributions}

CW and WZ designed the study. CW, WZ, and DBZ analyzed all the data. CW and DBZ wrote the main manuscript. YZ, WW, LZ, YYM, JF, and HCC abstracted data from the medical records. All authors read and approved the final manuscript.

\section{Acknowledgements}

Not applicable.

\section{References}

1. Vose J, Armitage J, Weisenburger D. International peripheral T-cell and natural killer/T-cell lymphoma study: pathology findings and clinical outcomes. J Clin Oncol. 2008;26(25):4124-30.

2. Sun J, Yang Q, Lu Z, et al. Distribution of lymphoid neoplasms in China: analysis of 4,638 cases according to the World Health Organization classification. Am J Clin Pathol. 2012;138(3):429-34.

3. Savage KJ, Chhanabhai M, Gascoyne RD, et al. Characterization of peripheral T-cell lymphomas in a single North American institution by the WHO classification. Ann Oncol. 2004;15(10):1467-75.

4. Sonnen R, Schmidt WP, Müller-Hermelink HK, et al. The International Prognostic Index determines the outcome of patients with nodal mature T-cell lymphomas. Br J Haematol. 2005;129(3):366-72.

5. Liu W, Ji X, Song Y, et al. Improving survival of 3760 patients with lymphoma: Experience of an academic center over two decades. Cancer Med. 2020 Apr 12. doi:10.1002/cam4.3037.

6. Abouyabis AN, Shenoy PJ, Sinha R, et al. A Systematic Review and Meta-Analysis of Front-line AnthracyclineBased Chemotherapy Regimens for Peripheral T-Cell Lymphoma. ISRN Hematol. 2011;2011:623924.

7. Ellin F, Landström J, Jerkeman M, et al. Real-world data on prognostic factors and treatment in peripheral T-cell lymphomas: a study from the Swedish Lymphoma Registry. Blood. 2014;124(10):1570-7.

8. Schmitz N, Trümper L, Ziepert M, et al. Treatment and prognosis of mature T-cell and NK-cell lymphoma: an analysis of patients with T-cell lymphoma treated in studies of the German High-Grade Non-Hodgkin Lymphoma Study Group. Blood. 2010;116(18):3418-25.

9. Gleeson M, Peckitt C, To YM, et al. CHOP Versus GEM-P in Previously Untreated Patients With Peripheral T-cell Lymphoma (CHEMO-T): A Phase 2, Multicentre, Randomised, Open-Label Trial. Lancet Haematol. 2015;(5):e190e200.

10. Yan Z, Wei Z, Jian L, et al. Gemcitabine, cisplatin, and dexamethasone (GDP) in combination with methotrexate and pegaspargase is active in newly diagnosed peripheral T cell lymphoma patients: a phase 2, single-center, open-label study in China. Ann Hematol. 2019;98:143-50.

11. Piekarz RL, Frye R, Prince HM, et al. Phase 2 trial of romidepsin in patients with peripheral T-cell lymphoma. Blood. 2011;117(22):5827-34. 
12. O'Connor OA, Horwitz S, Masszi T, et al. Belinostat in Patients With Relapsed or Refractory Peripheral T-Cell Lymphoma: Results of the Pivotal Phase II BELIEF (CLN-19) Study. J Clin Oncol. 2015;33(23):2492-9.

13. Lu X, Ning Z, Li Z, Cao H, Wang X. Development of chidamide for peripheral T-cell lymphoma, the first orphan drug approved in China. Intractable Rare Dis Res. 2016;5(3):185-91.

14. Shi Y, Dong M, Hong X, Zhang W, Feng J, Zhu J, et al. Results from a multicenter, open-label, pivotal phase II study of chidamide in relapsed or refractory peripheral T-cell lymphoma. Ann Oncol. 2015;26(8):1766-71.

15. Shi Y, Jia B, Xu W, et al. Chidamide in relapsed or refractory peripheral T cell lymphoma: a multicenter real-world study in China. J Hematol Oncol. 2017 Mar;15(1):69. 10(.

16. Swerdlow SH, Campo E, Harris NL, et al. WHO Classification of tumours of haematopoietic and lymphoid tissues. 4th ed. Vol. 2. Lyon: International Agency for Research on Cancer; 2008.

17. A predictive model for aggressive non-Hodgkin's lymphoma. The International Non-Hodgkin's Lymphoma Prognostic Factors Project. N Engl J Med. 1993;329(14):987-94.

18. Gallamini A, Stelitano C, Calvi R, et al. Peripheral T-cell lymphoma unspecified (PTCL-U): a new prognostic model from a retrospective multicentric clinical study. Blood. 2004;103(7):2474-9.

19. Cheson BD, Pfistner B, Juweid ME, et al. International Harmonization Project on Lymphoma. Revised response criteria for malignant lymphoma. J Clin Oncol. 2007;25:579-86.

20. Juweid ME, Stroobants S, Hoekstra OS, et al. Imaging Subcommittee of International Harmonization Project in Lymphoma. Use of positron emission tomography for response assessment of lymphoma: consensus of the Imaging Subcommittee of International Harmonization Project in Lymphoma. J Clin Oncol. 2007;25(5):571-8.

21. Mercadal S, Briones J, Xicoy B, et al. Intensive chemotherapy (high-dose CHOP/ESHAP regimen) followed by autologous stem-cell transplantation in previously untreated patients with peripheral T-cell lymphoma. Ann Oncol. 2008;19(5):958-63.

22. Escalón MP, Liu NS, Yang Y, et al. Prognostic factors and treatment of patients with T-cell non-Hodgkin lymphoma: the M. D. Anderson Cancer Center experience. Cancer. 2005;103(10):2091-8.

23. Dupuis J, Morschhauser F, Ghesquières $\mathrm{H}$, et al. Combination of romidepsin with cyclophosphamide, doxorubicin, vincristine, and prednisone in previously untreated patients with peripheral T-cell lymphoma: a nonrandomised, phase 1b/2 study. Lancet Haematol. 2015;2(4):e160-5.

24. d'Amore F, Relander T, Lauritzsen GF, et al. Up-front autologous stem-cell transplantation in peripheral T-cell lymphoma: NLG-T-01. J Clin Oncol. 2012;30(25):3093-9.

25. Horwitz S, O'Connor OA, Pro B, et al. Brentuximab vedotin with chemotherapy for CD30-positive peripheral T-cell lymphoma (ECHELON-2): a global, double-blind, randomised, phase 3 trial. Lancet. 2019;393(10168):229-40.

26. Gisselbrecht C, Gaulard P, Lepage E, et al. Prognostic significance of T-cell phenotype in aggressive nonHodgkin's lymphomas. Groupe d'Etudes des Lymphomes de l'Adulte (GELA). Blood. 1998;92(1):76-82.

27. Sonnen R, Schmidt WP, Müller-Hermelink HK, et al. The International Prognostic Index determines the outcome of patients with nodal mature T-cell lymphomas. Br J Haematol. 2005;129(3):366-72.

28. Kim SJ, Kim JH, Ki CS, et al. Epstein-Barr virus reactivation in extranodal natural killer/T-cell lymphoma patients: a previously unrecognized serious adverse event in a pilot study with romidepsin. Ann Oncol. 2016;27(3):50813.

\section{Figures}

Page $13 / 14$ 

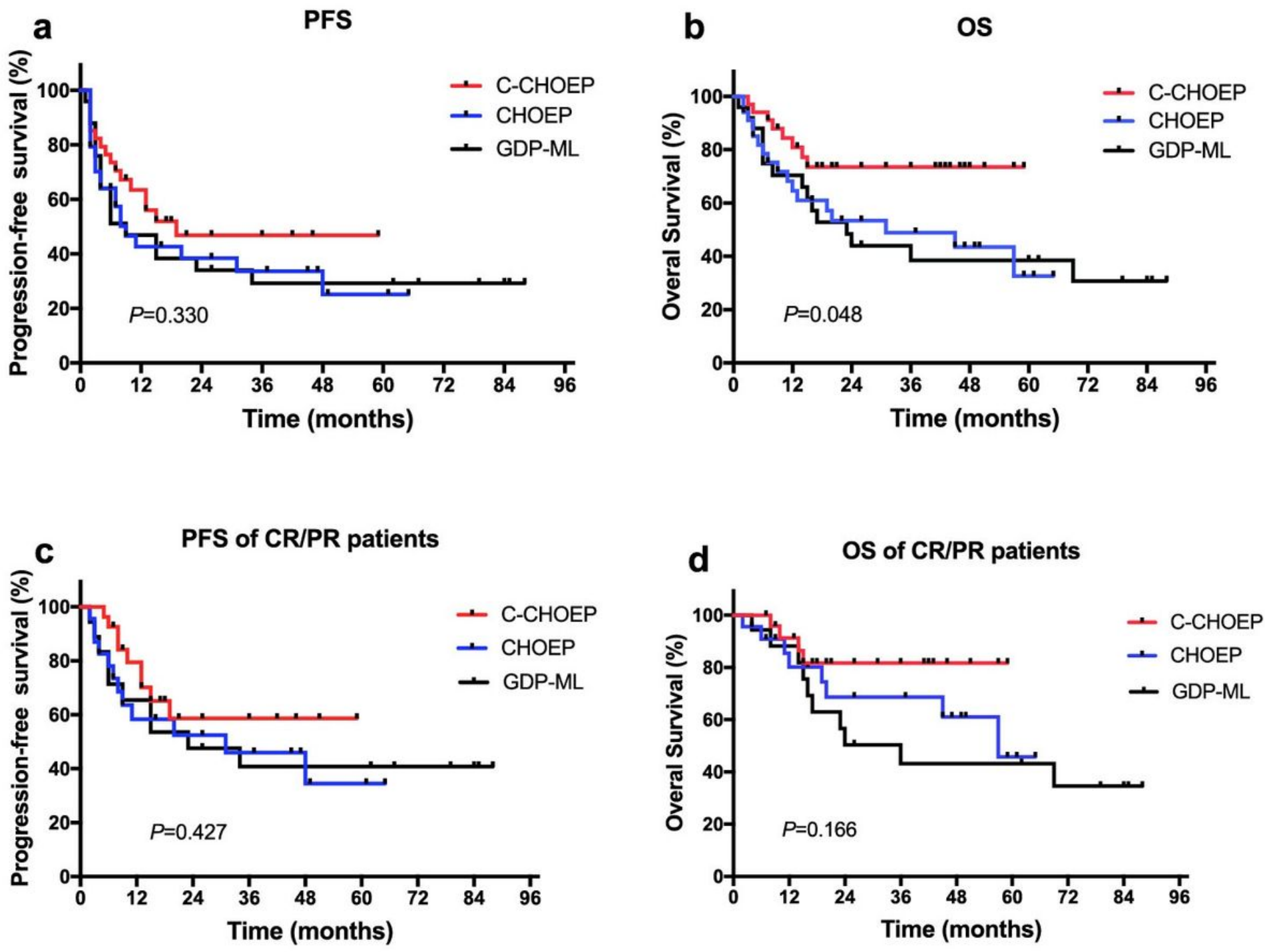

Figure 1

Kaplan-Meier curves of PFS for all patients (a) and responding patients (c) in the 3 groups, OS for all patients (b) and responding patients (d) in the 3 groups 